\title{
Large-scale protein/antibody patterning with limiting unspecific adsorption
}

\author{
Viktoriia Fedorenko ${ }^{1,2}$, Mikhael Bechelany ${ }^{1}$, Jean-Marc Janot ${ }^{1}$, Valentyn Smyntyna ${ }^{2}$, Sebastien \\ Balme $^{1 *}$
}

${ }^{1}$ Institut Européen des Membranes IEMM, ENSCM, UM, CNRS, UMR5635, Place Eugène Bataillon, F-34095 Montpellier Cedex 5, France

${ }^{2}$ Faculty of Physics, Experimental physics department, Odessa National I. I. Mechnikov University, 42, Pastera, 65026, Odessa, Ukraine

*Corresponding author : sebastien.balme@umontpellier.fr

\begin{abstract}
A simple synthetic route based on nanosphere lithography has been developed in order to design largescale nanoarray for specific control of protein anchoring. This technique based on two-dimensional (2D) colloidal crystals composed of polystyrene spheres allows the easy and inexpensive fabrication of large arrays (up to several centimeters) by reducing the cost. A silicon wafer coated with a thin adhesion layer of chromium $(15 \mathrm{~nm})$ and a layer of Gold $(50 \mathrm{~nm})$ is used as a substrate. PS spheres are deposited on the gold surface using the floating-transferring technique. The PS spheres were then functionalized with PEG-biotin and the defects by self-assembly monolayer (SAMs) PEG to prevent unspecific adsorption. Using epifluorescence microscopy, we show that after immersion of sample on target protein (avidin and anti-avidin) solution, the latter are specifically located on polystyrene spheres. Thus, these results are meaningful for exploration of devices based on large-scale nanoarray of PS spheres and can be used for detection of target proteins or simply to pattern a surface with specific proteins.
\end{abstract}




\section{Introduction}

In the area of biosensing and bioengineering, one of the challenges is to produce large-scale patterning for specific anchoring of a target protein. This challenge requires an ability to manipulate nanoobjects, to order them on large surface areas and to control the location of protein attachment. One of the strategies for the deposition of a large scale array is the nanosphere lithography using twodimensional (2D) colloidal crystals that is composed of microscale polystyrene sphere (PS spheres). This technique as introduced by Deckman and Dunsmuir (Deckman and Dunsmuir 1982) allows the synthesis of large arrays (several millimeters). (Sakamoto, Philippe et al. 2008, Bechelany, Brodard et al. 2009) This method is fascinating because it is rather easy in comparison to the traditional lithographical methods. Using this process, well ordered and controlled micro/nanostructures could be designed on a large area substrate using a mono or several layers of PS spheres.

Polystyrene is one of the most extensively used types of plastic and the number of its applications is growing over the past 20 years. It is an aromatic polymer obtained by polymerization of styrene monomers (Wünsch 2000). It is also biocompatible and is not expected to adversely affect interactions of nanoparticles with biological systems. Polystyrene nanoparticles have been used to fashion largescale nanoarray for various applications, such as biosensors (Velev and Kaler 1999), photonics (Rogach, Susha et al. 2000), and self-assembling of nanostructures (Boal, Ilhan et al. 2000). Specifically surface-modified polystyrene nanoparticles are homogeneous, exhibit a low polydispersity index, and form stable colloids in biological fluids (Florence 2004). Because polystyrene nanoparticles can be easily synthesized in a wide range of sizes with distinct surface functionalization, they are perfectly suited as model particles to study the effects of the particle surface characteristics on various biological parameters. The protein micro/nano patterning using PS spheres has been previously reported. In most of case, the strategy consists to remove the sphere in order to adsorb the proteins. (Wang, Yap et al. 2005, Mallon, Jose et al. 2010, Taylor, Keay et al. 2012) There are several limitations for this strategy because the adsorption is an unspecific process which is not suitable to develop specific biosensor. This process does not allow controlling the protein orientation and denaturation. 
Thus the development of large-scale nanoarray requires an effort to control the protein location, integrity and selectivity; this includes preventing the unspecific adsorption and the grafting of specific probe on the surface. The adsorption process is regulated by the properties of the protein as well as those of the surface of the material, the environmental conditions and the kinetics of the process.(Balme, Janot et al. 2006, Balme, Coulon et al. 2016) In the case of biosensor, unspecific protein adsorption may limit the effectiveness of the surface that provides the active component of the biosensors. This induces a low signal from the sensor and often causes high background (noise) signal. Thus, the control of the adsorption process is even more critical for applications that use nanostructured substrates with large relative surface areas, such as nanoparticles, microchannels, and thin porous membranes, due to their large surface.

There are different strategies to prevent unspecific adsorption of proteins, such as coating by bovine serum albumin (BSA), grafting of phospholipids, use of non-ionic detergents like Tween 20 or of polymeric materials like polyethylene glycol (PEG), as well as combinations of these elements (Washburn, Luchansky et al. 2009, Bog, Brinkmann et al. 2015). PEG is one of the most-studied materials used in order to obtain antibioadherent coatings, especially in the pharmaceutical, cosmetic, and biomedical fields (Hamming and Messersmith 2008, Obermeier, Wurm et al. 2011, Soteropulos, Zurick et al. 2012). The interest of this polymer is due to its unique physical and chemical properties. Indeed it is highly hydrophilic and has significant chain flexibility (Uchida, Otsuka et al. 2005). It has also excellent solubility in aqueous and organic and has a low toxicity to living cells (Zalipsky and Milton Harris 1997). Preventing unspecific adsorption via its strong hydration layer and its steric stabilization effect is one of the most widespread applications of PEG. (Zalipsky and Milton Harris 1997, Chapman, Ostuni et al. 2000). This coating by PEG is used to control the interaction of biological molecules with the surface of biosensors. This functionalization is commonly done by thiolPEG self-assembled monolayers (SAM) on gold surface. (Ostuni, Chapman et al. 2001, Ma, Hyun et al. 2004, Gudipati, Finlay et al. 2005).

The SAMs produced on various substrates have been extensively investigated for the surfaces functionalization with active molecules or for protecting them from adsorption of species present in the environment (Ulman 1996, Aswal, Lenfant et al. 2006). The SAMs formation is induced by the 
strong chemisorption between the substrate and the head group of the selected organic molecules. This allows making stable ultrathin organic films with controlled thickness (Bain and Whitesides 1989, Dubois and Nuzzo 1992). These advantages make the SAMs particularly suited to the development of applications in the field of biosensors (Love, Estroff et al. 2005).This makes them an ideal model in many areas such as bioanalytical, organometallic, physical organic, bioorganic and electrochemistry (Dubois and Nuzzo 1992, Bishop and Nuzzo 1996, Ulman 1996). Usually, SAMs formed on gold material or surface are attached using thiol-modified molecules. The self-assembled monolayers of alkanethiols on gold are probably one of the best surfaces currently available to obtain the functionalization and the patterning of biosensors with advantages such as flexibility and stability. The gold-thiol monolayers are stable when exposed to air, to water or ethanolics solutions for several months (Bain, Troughton et al. 1989). They are also quite easy to produce, quickly assembled and well ordered.

This work aims to produce a large surface of nanoarray functionalized with proteins for antibody detection. Herein, we report the synthesis of multifunctional surface with polystyrene spheres and selective functionalization ability. We aim to attach specifically the protein directly to the PS spheres and to limit its unspecific adsorption. In our work, we used a silicon wafer coated with a thin adhesion layer of chromium $(15 \mathrm{~nm})$ and a layer of noble metal $(50 \mathrm{~nm})$. PS spheres are deposited on the gold surface using the floating-transferring technique. We design a multifunctional surface with array of polystyrene spheres. We used PEG SAMs to block the unspecific adsorption of protein on the surface of these polystyrene spheres. Another interesting point is relative to the stability of PS spheres layer regarding the multisteps functionalization which could create defects on the surface.

\section{Materials and method}

\subsection{Materials}

Avidin from egg white (A9275), Poly(ethylene glycol) 2-aminoethyl ether biotin (PEG) $M_{n}=5300 \mathrm{~g}$ $\mathrm{mol}^{-1}$ (757772), N-Hydroxysuciinimide (NHS) $\mathrm{Mw}=115.09 \mathrm{~g} \mathrm{~mol}^{-1}$ (130672), Ethylenediamine Mw $=60.10 \mathrm{~g} \mathrm{~mol}^{-1}$ (E26266), Anti-Avidin, antibody produced in rabbit (A5170), Ethanol (absolute 
alcohol, without additive (02860)), phosphate buffered saline (P4417) and Microparticles based on polystyrene, (size $1 \mu \mathrm{m}, 10 \mathrm{wt} \%$ aqueous solution (89904)) were purchased from Sigma Aldrich. Thiol PEG, mPEG-SH Mw = $2000 \mathrm{~g} \mathrm{~mol}^{-1}(\mathrm{PG} 1-\mathrm{TH}-2 \mathrm{k})$, Cy5-PEG-NHS Mw $=5000 \mathrm{~g} \mathrm{~mol}^{-1}(\mathrm{PG} 2-\mathrm{NSS} 5-$ 5k) and Succinimidyl PEG, mPEG-NHS Mw = $2000 \mathrm{~g} \mathrm{~mol}^{-1}$ (PG1-SC-2k) were purchased from Nanocs. N-(3-Dimethylaminopropyl)-N-ethylcarbodiimide hydrochloride (EDC) $\mathrm{Mw}=191.70 \mathrm{~g} \mathrm{~mol}^{-1}$ (03449) was purchased from Fluka Analytical. Alexa Fluor 488 TFP ester (A37570) and Alexa Fluor 647 NHS (A37573) were purchased from ThermoFisher Scientific.

\subsection{Protein labeling:}

Avidin was labeled with Alexa Fluor 647 NHS. Typically, $100 \mu \mathrm{L}$ of protein solution $(1 \mathrm{mg}$ Avidin and $100 \mu \mathrm{l}$ PBS $\mathrm{pH}=8.3$ ) was added to dry fluorophore Alexa-647 and allowed to react for $0.5 \mathrm{~h}$ at 20 ${ }^{\circ} \mathrm{C}$. Anti-Avidin was labeled using AlexaFluor488 TFP ester. $100 \mu \mathrm{L}$ of protein solution $(5 \mu \mathrm{L}$ AntiAvidin and $100 \mu \mathrm{lBS} \mathrm{pH}=8.3$ ) was added to dry fluorophore Alexa-488 and allowed to react for 0.5 $\mathrm{h}$ at $20{ }^{\circ} \mathrm{C}$. Then the unreacted dyes and proteins were separated by centrifugation $(16000 \mathrm{~g}, 1$ min) using filter (Biospin P6) according to the supplier of the kit.

\subsection{Polystyrene sphere deposition on the Si/Cr/Au surface}

The nanoarrays of polystyrene spheres were designed using the following procedure. A deposition of $15 \mathrm{~nm}$ of $\mathrm{Cr}$ and $50 \mathrm{~nm}$ of Au were performed on p-type $\mathrm{Si}(100)$ wafers $\left(2 \times 2 \mathrm{~cm}^{2}\right.$ pieces of boron doped $(8-25 \Omega \mathrm{cm})$ ). The substrates were treated by $\mathrm{O}_{2}$ plasma using a pressure of $0.6 \mathrm{mbar}$ and a power supply of $0.15 \mathrm{~A}$ in order to eliminate all the organic matters from the surface. The polystyrene spheres (PS spheres) were then deposited using the floating transferring technique.

Typically, the monolayers of spheres were prepared using monodisperse polystyrene particles with the diameter of $1 \mu \mathrm{m} .40 \mu \mathrm{l}$ of the polystyrene solution (10 wt $\%$ ) was diluted by an equal amount of ethanol. The solution was applied onto the $\mathrm{Si}$ substrate and displayed over the $\mathrm{Si}$ surface. The $\mathrm{Si}$ substrate was maintained stationary for few seconds in order to allow a good dispersion of the PS spheres. The Si wafer was then slowly immersed into a glass vessel filled with deionized water (80 
ml). A non-ordered monolayer of PS spheres was formed on the water surface. A droplet of $10 \%$ sodium dodecyl sulfate (SDS) solution was added to the water in order to modify the surface tension. This step targets to consolidate the PS spheres. A large monolayer of PS spheres with highly ordered areas was achieved. This monolayer of PS spheres was transferred to our target substrate $(\mathrm{Si} / \mathrm{Cr}(15$ $\mathrm{nm}) / \mathrm{Au}(50 \mathrm{~nm}))$. After drying, the $\mathrm{Si}$ substrate covered with the PS was heated at $100{ }^{\circ} \mathrm{C}$ for $10 \mathrm{~min}$ in order to sinter the PS spheres and to improve their adhesion into the Si substrate. After sintering, the diameter of the PS spheres was reduced with etching by $\mathrm{O}_{2}$ plasma for different times: $1,5,10$ and 15 $\min$. In this experiment, the utilized pressure of $\mathrm{O}_{2}$ plasma is 0.6 mbar and the power supply is $0.15 \mathrm{~A}$.

\subsection{Functionalization of nanoarrays}

The PEGylating of gold surface by SAMS: The nanoarray was placed into $1 \mathrm{ml}$ of solution of thiol PEG, (mPEG-SH) (4 mg) in unmodified ethanol for two days. Then the samples were rinsed with ethanol.

Polystyrene sphere biotinylation: After PEGylation by SAMS, the nanoarrays were placed in a solution of $0.5 \mathrm{mg} \mathrm{mL}^{-1}$ PEG-biotin- $\mathrm{NH}_{2}, 2 \mathrm{mg} \mathrm{mL}^{-1} \mathrm{EDC}$ and $1 \mathrm{mg} \mathrm{mL}^{-1} \mathrm{NHS}$ for 24 hours. After this period, the sample is rinsed with ethanol to remove the absorbed PEG.

mPEG-NHS and Cy5-PEG-NHS grafting on polystyrene sphere: In order to link mPEG-NHS or Cy5-PEG-NHS on polystyrene surface, we have firstly grafted Ethylenediamine on $\mathrm{COOH}$ groups as follows:(i) the nanoarrays were immersed in $1 \mathrm{ml}$ ethanol solution which contained $2 \mathrm{mg} \mathrm{mL}^{-1} \mathrm{EDC}$ and $1 \mathrm{mg} \mathrm{mL} \mathrm{m}^{-1} \mathrm{NHS}$ for 2 hours. (ii) Then the sample is rinsed with ethanol, and it is placed in solution of mPEG-NHS or Cy5-PEG-NHS $\left(0.2 \mathrm{mg} \mathrm{mL}^{-1}\right)$ for one hour. (iii) Finally, the samples were rinsed with ethanol.

Protein attachment assays: For protein attachment assay, we used Avidin, Avidin labeled with Alexa Fluor 647 NHS (noted Avidin*) and Anti-Avidin labeled with AlexaFluor488 TFP ester (noted AntiAvidin*). The nanoarrays were immersed for $30 \mathrm{~min}$ in $1 \mathrm{ml}$ of protein $\left(10 \mu \mathrm{g} \mathrm{ml}^{-1}\right)$ in PBS $(0.01 \mathrm{M}$ phosphate buffer, $0.0027 \mathrm{M}$ potassium chloride and $0.137 \mathrm{M}$ sodium chloride, $\mathrm{pH} 7.4$, at $25^{\circ} \mathrm{C}$ ). Then the samples were rinsed with PBS. 


\subsection{Microscopy}

The morphology of the samples was observed with scanning electron microscopy (SEM, Hitachi S4800). Fluorescence images were performed using an epifluorescence microscope DM6000. The alexa fluor 647 and 488 were detected using CY5 and FTIC cube respectively.

\section{Results and discussion}

The present work aims to produce large-scale nanoarrays which permit to control protein grafting and limit the unspecific adsorption. The large-scale nanoarrays can be produced by nanospheres lithography using the floating transferring technique of polystyrene sphere. These spheres can serve as anchor site for protein attachment. However, the main limitation comes from the unspecific adsorption between the spheres. In order to tackle this limitation, one way is the functionalization of these free spaces with a high density of PEG molecules. This can be achieved by Thiol PEG SAMs strategies on gold surface since it is well-known strategy to limit the non-specific adsorption of protein. The overall process for nanoarrays design is presented on the schematic diagram (Figure 1).

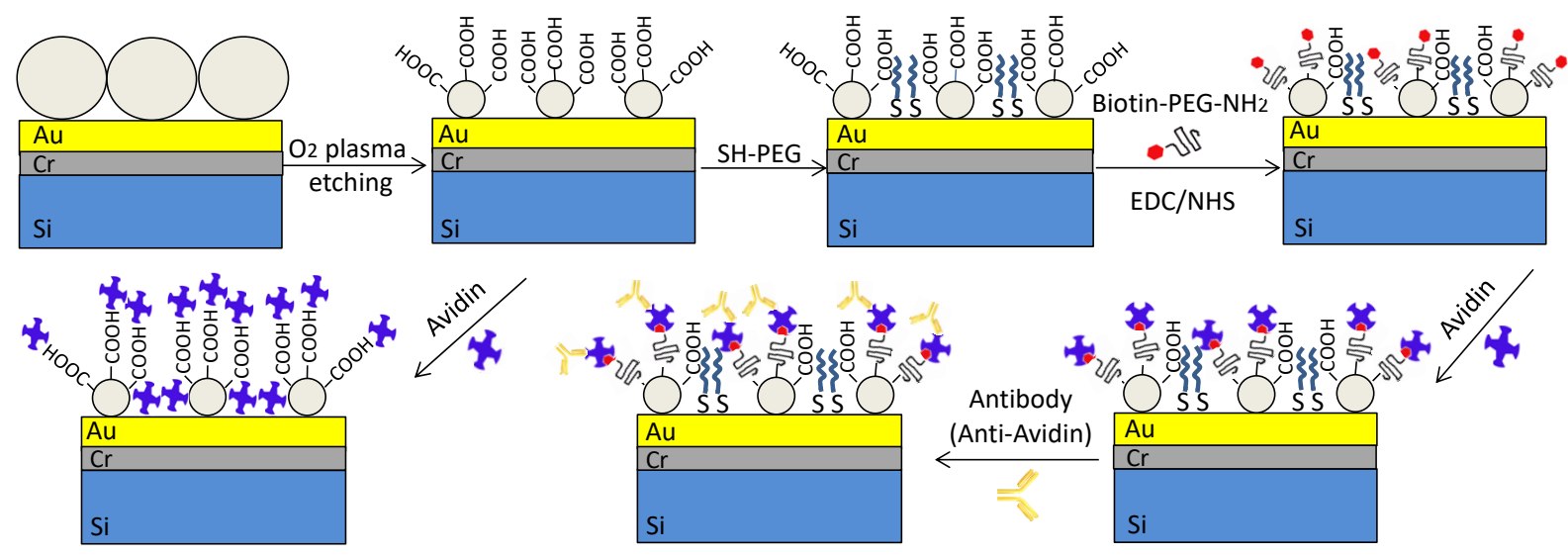

Figure 1. The schematic diagram of overall process for protein immobilization

The first step consists to deposit a thin layer of gold $(50 \mathrm{~nm})$ on $\mathrm{Si}$ water. In order to improve the stability of this layer, $15 \mathrm{~nm}$ of $\mathrm{Cr}$ is deposited on the $\mathrm{Si}$ substrate before the Au deposition. The 
$\mathrm{Si} / \mathrm{Cr} / \mathrm{Au}$ substrates were treated by $\mathrm{O}_{2}$ plasma to remove all organic matter from the surface. After the pretreatment, an ordered monolayer of polystyrene spheres (PS spheres) was prepared and deposited on the substrate by floating transferring technique(Rybczynski, Hilgendorff et al. 2003, Mikhael, Elise et al. 2011). The sample was prepared as described in the experimental section and dried in air at room temperature. The obtained spheres were self-assembled into a close-packed two-dimensional ordered area via attractive capillary forces (Danov, Pouligny et al. 2001). After drying, Si substrate covered with PS was heated in an oven at $100{ }^{\circ} \mathrm{C}$ for $10 \mathrm{~min}$ to sinter the PS and to improve their adhesion to the $\mathrm{Si} / \mathrm{Cr} / \mathrm{Au}$ substrate. The diameter of spheres was decreased with etching by $\mathrm{O}_{2}$ plasma. This step permits also as well to oxidize the polystyrene surface to create $\mathrm{COOH}$ group on their surface. The latter step is essential for further functionalization of PS spheres. The SEM image (Figure 2) of PS spheres shows the unformal decrease of the diameter with the plasma treatment. In addition, it confirms that the transfer permit to obtain the monolayer of PS spheres. The dependence mean diameter of PS spheres as the function of different times of etching by $\mathrm{O}_{2}$ plasma is also reported on Figure 2. The characterization of protein and Antiboby on the nanoarrays is performed by epifluorescence microscopy. The minimum size requires to detect the PS spheres is around $900 \mathrm{~nm}$. Thus we have fixed etching time to 5 minutes. This time permits to decrease the PS spheres diameter from $956 \mathrm{~nm}$ to $933 \mathrm{~nm}$. 


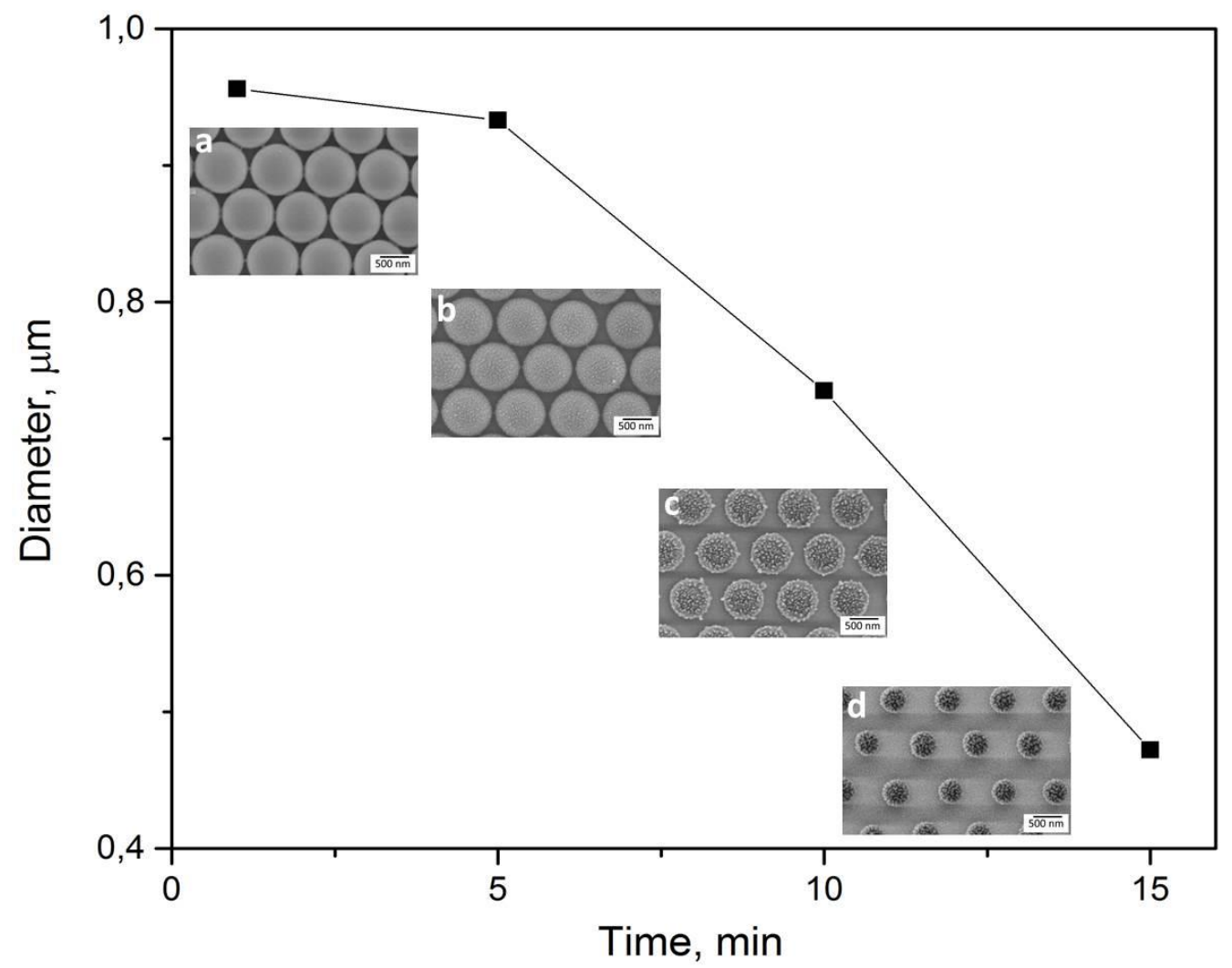

Figure 2. Diameter of PS spheres as the function of the $\mathrm{O}_{2}$ plasma etching: The insets show PS spheres with different $\mathrm{O}_{2}$ plasma etching times: 1 (a), 5 (b), 10 (c) and 15 (d) min, respectively, as determined by SEM (scale bars $500 \mathrm{~nm})$.

After the plasma etching, the sample is composed by zone. The first one is the gold metallic surface which is uncovered by polystyrene spheres. The second one is the surface of PS spheres with exhibits $\mathrm{COOH}$ group. These two parts of the sample could be functionalized differently because we expect to graft protein and antibody specifically on PS spheres. This imposes to prevent the absorption between the PS spheres. To do it, the gold surface between PS spheres was functionalized by SAMS of PEGthiol.

After this step, we are able to have two different functionalizations on spheres and between them on the gold surface. We attached on polystyrene spheres the PEG-Cy5 following two steps. The first one consists to link ethylene diamine on carboxyl groups using EDC/NHS, and the second one is the addition of NHS-PEG-Cy5. Figure 3a shows a fluorescence microscopy image of the prepared sample. 
We can observe that fluorescence came only from the spheres and there is no fluorescence between them. The diameter of spheres around $0.9 \mu \mathrm{m}$ is in good agreement with the one determined by SEM. Several additional, experiments were performed as blank to confirm that the fluorescence comes from the dyes. PS spheres do not exhibit self-fluorescence under excitation wavelength of 590-650 nm provided by Cy5 cube. In addition the images performed after each step of functionalization (after reduction by plasma, gold coating and PEG-biotin grafting) do not reveal fluorescence emission under the same condition. A weak autofluorescence of PS sphere was only observed under excitation wavelength lower than $450 \mathrm{~nm}$ (using Hoeechst and CFT cubes).According to that, we can conclude that the PEG-Cy5 is attached on PS spheres. Between the PS spheres, we observe some defects. However, PS spheres cover the majority of the surface of the sample (here $90.48 \times 67.6 \mu \mathrm{m}^{2}$ ). At this step, our results prove that our strategy allows designing a large-scale of monolayer of PS spheres and that our strategy of functionalization is relevant to functionalize PS spheres surface and gold surface with different functions.

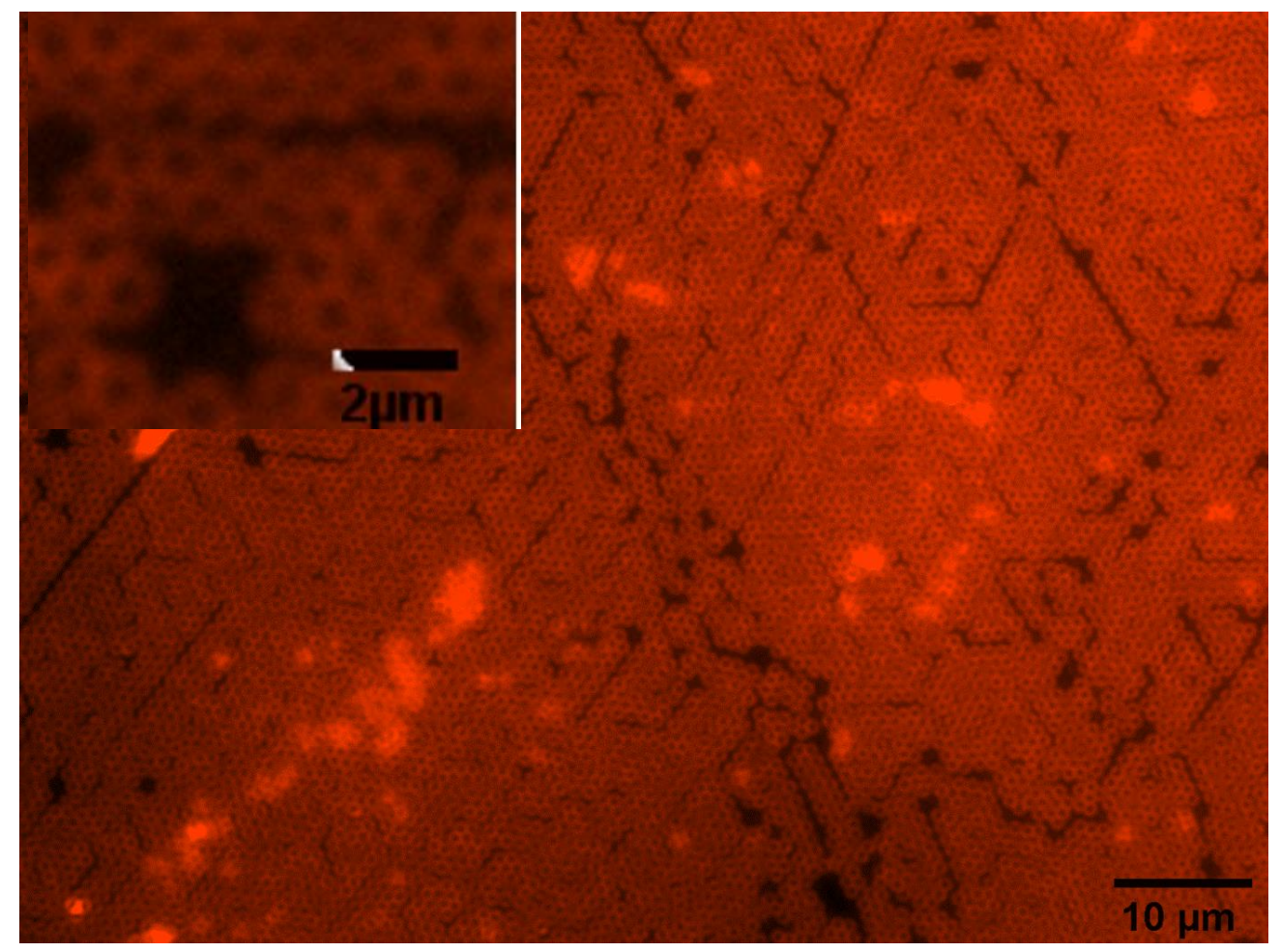

Figure 3. Fluorescent images of Cy5-PEG-NHS-modified polystyrene spheres (In inset a zoom) 
We wanted to use similar strategy to attach the protein directly to the spheres, but not between them. To do it, we have done a variety of experiments using the avidin-biotin strategy. The latter is commonly used to design biosensor device. The first step consists to graft PEG-biotin on PS spheres. This was performed using PEG-biotin- $\mathrm{NH}_{2}$ and EDC/NHS. In the second step, the avidin labelled with Alexa647 was added and their localization was investigated by epifluorescence spectroscopy (Figure 4). As expected, the fluorescence comes specifically from the PS spheres. The lake of fluorescence between the spheres proves that there is no- protein. Thus the functionalization of gold surface is efficient to prevent the unspecific adsorption of protein. In order to prove that the avidin is on PS spheres due to the presence of biotin, a similar functionalization with mPEG on PS spheres was performed. After immersion, under labelled avidin, the epifluorescence reveals a weak fluorescence. Since, the PS spheres and the functionalization do not reveal fluorescence emission using CY5 cube, we can admit that the signal is due to avidin likely adsorbed on sphere. Actually this is not surprising because PEG layer has to be dense to prevent the protein adsorption. In the case of PS spheres, the PEGs are grafted on carboxylate group. Thus, the density of these groups is likely too weak to induce a dense layer of PEG. In this case, proteins could be adsorbed on the defects and/or between the PEG chains. 

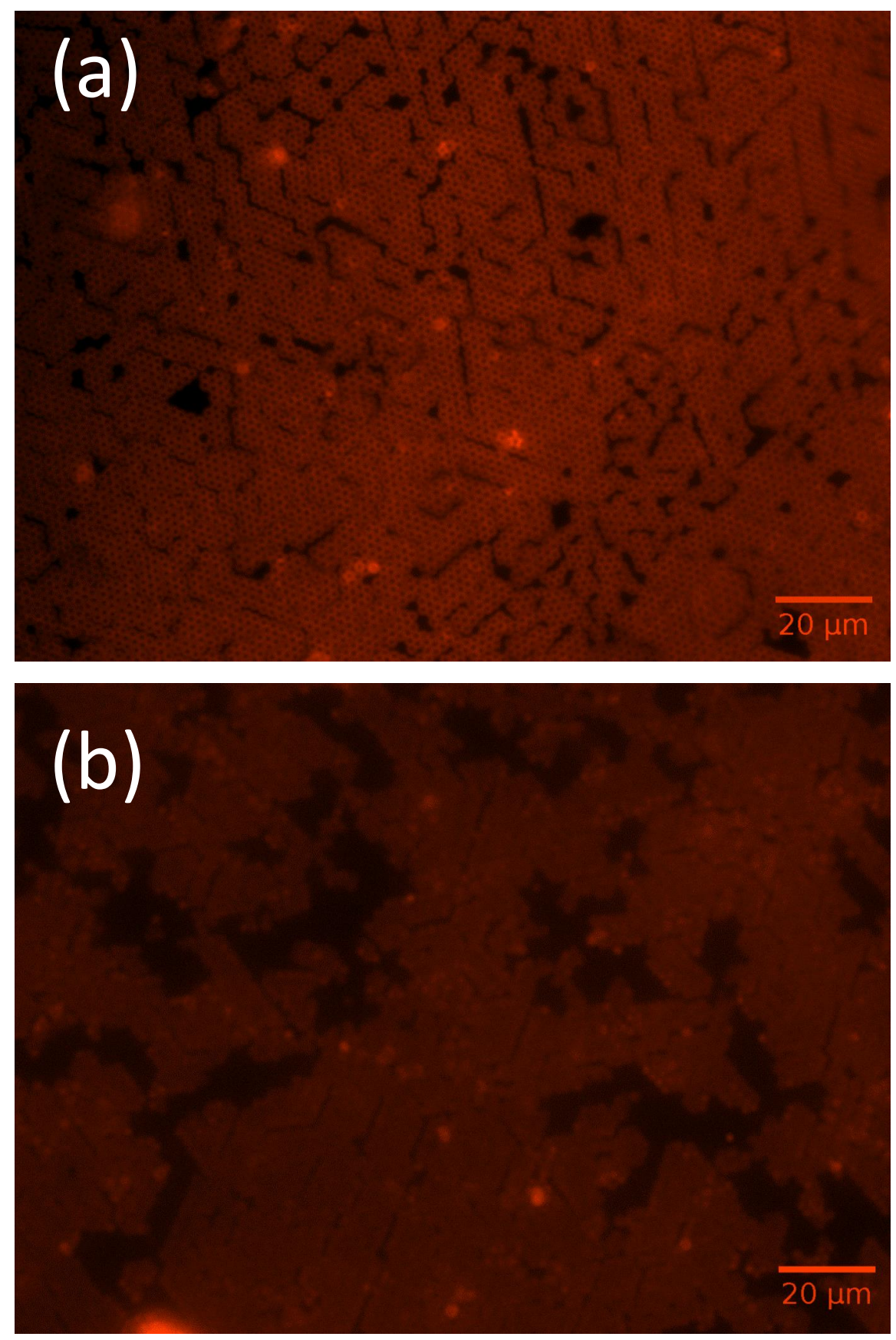

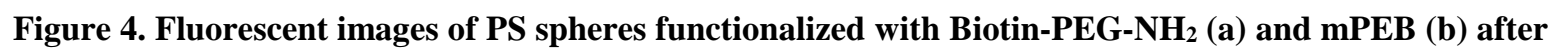
addition of avidin (labeled with alexa fluor 647)

In order to prove that the large-scale nanoarrays are suitable for antibody detection, different samples were functionalized with PEG-biotin and unlabeled avidin. After this first step, no fluorescence can be detected under excitation wavelength of $460-500 \mathrm{~nm}$ as well as $590-650 \mathrm{~nm}$. Then, they were immersed in solution containing Anti-Avidin* labelled with alexafluor 488. As expected, the epifluorescence image shows that anti-avidin is specifically located on PS spheres (Figure 5). The 
functionalization with SAMs of PEG prevents the antiboby as well as the avidin adsorption. We can observe that some microspheres have different intensity of green fluorescence. This could be explained by : (i) the number of dyes on antibodies is not homogeneous or (ii) the number of antibodies attached on microspheres is different.

The Figure 6 despites the fluorescence image of a sample where both avidin* and anti-avidin* are labeled with alexa fluor 647 and 488 respectively. The first one is characterized with CY5cube and the second one with FTIC cube. The perfect superposition of both images confirms that both avidin and anti-avidin are located on PS spheres.

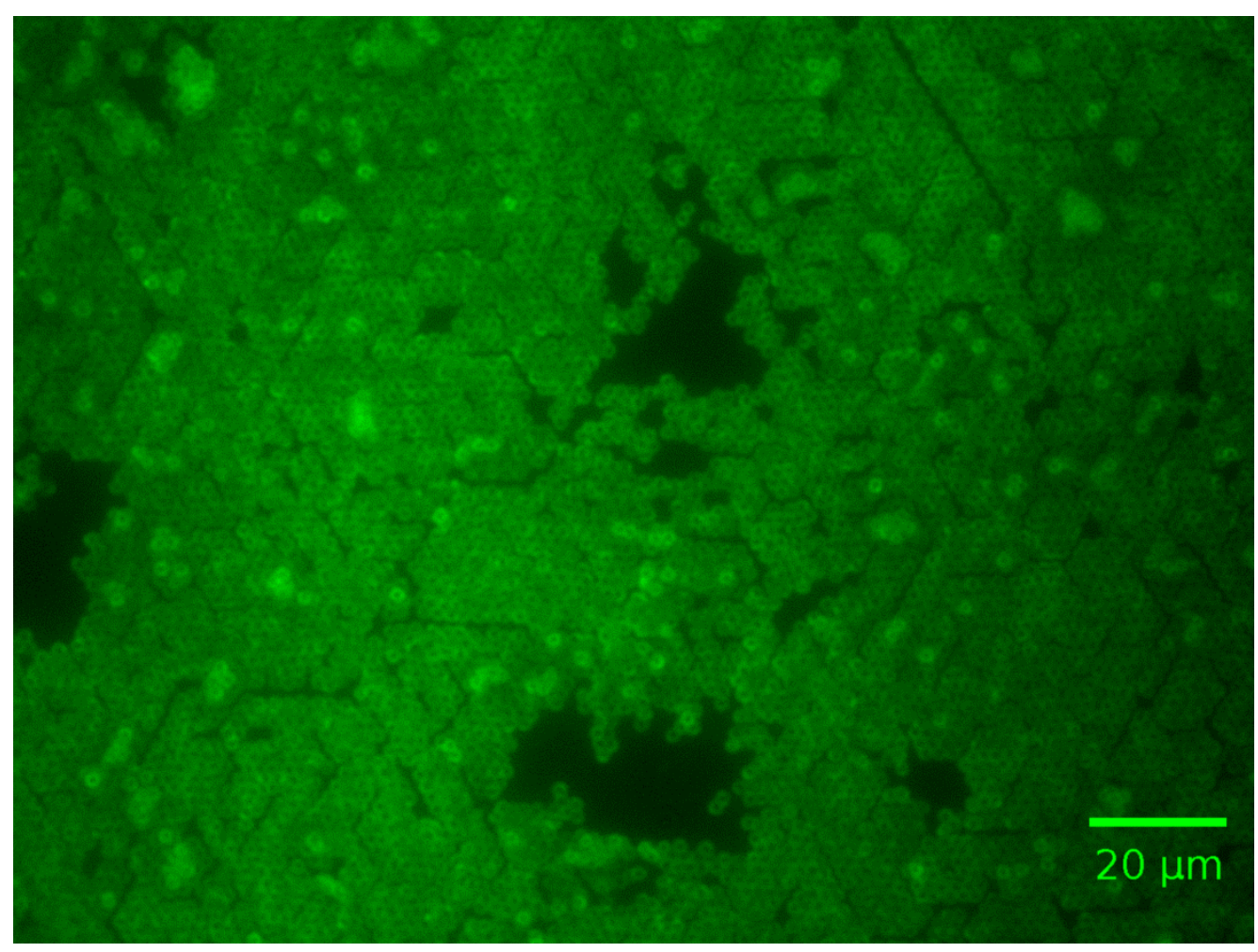

Figure 5. Fluorescent images of PS spheres functionalized with Biotin-PEG-NH 2 and avidin (unlabelled) after AntiAvidin*(labeled with Alexafluor 488) 

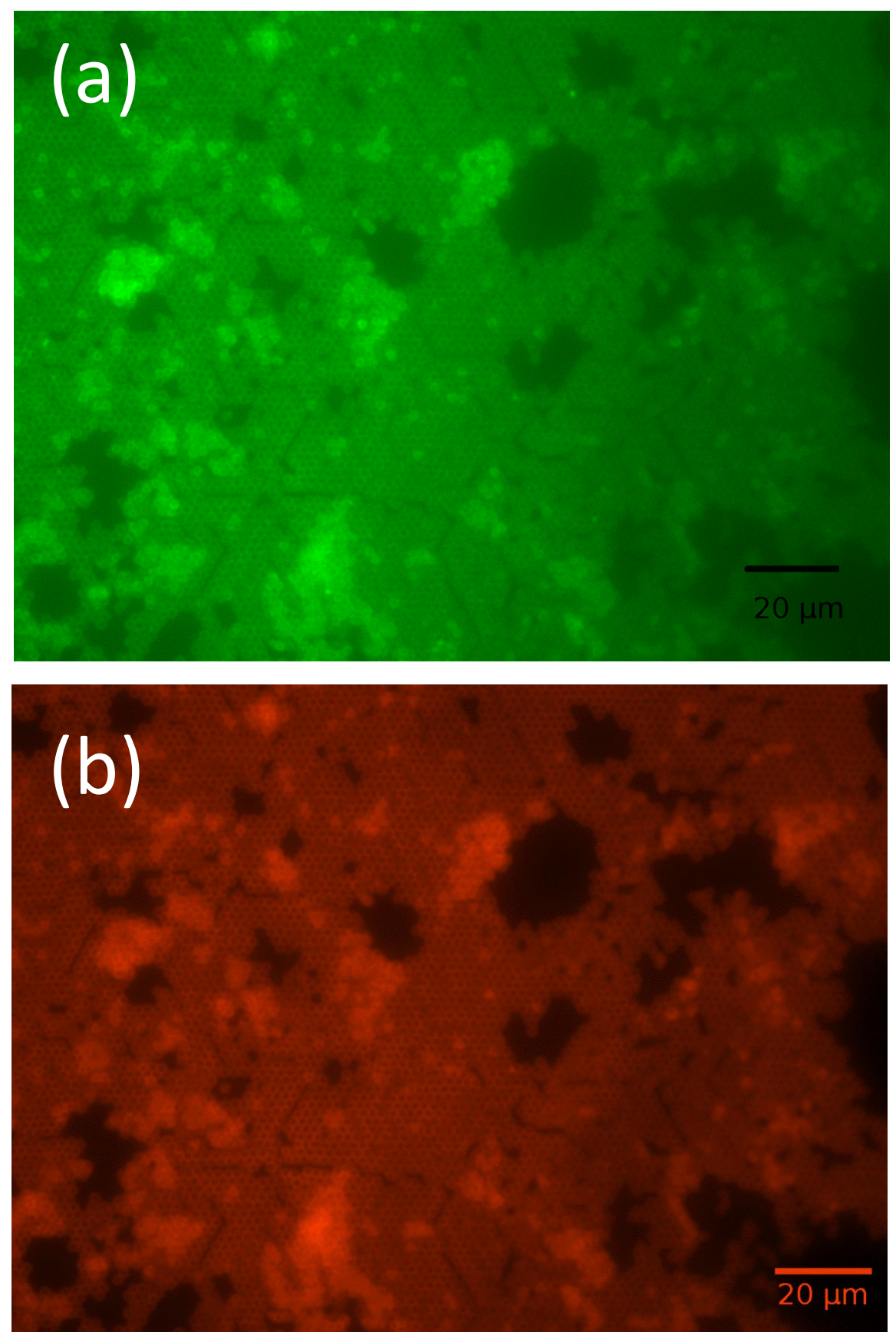

Figure 6 : Fluorescent images of PS spheres functionalized with Biotin-PEG-NH 2 and avidin (labeled with alexa fluor 647) after AntiAvidin (labeled with Alexafluor 488) addition recorded with FTIC cube (a) Cy5cube (b)

\section{Conclusion}

In summary, we have presented a novel method based on nanosphere lithography in order to design large-scale protein patterning. This technique based on two-dimensional (2D) colloidal crystals (polystyrene sphere (PS spheres)) allows the fast fabrication of large arrays (up to several centimeters) 
by reducing the cost. The nanoarrays are formed by a monolayer of polystyrene spheres deposit by floating-transferring technique on gold surface. On one hand, the gold surface between spheres was functionalized with SAMs of PEG to prevent the unspecific adsorption. On the other hand, the PS spheres were functionalized with PEG biotin. After addition of avidin and anti-avidin, the proteins are located only on PS spheres confirming that our strategy is suitable to control their location. Our results are meaningful for exploration of devices based on large-scale protein patterning on PS spheres. Regarding the versatility of avidin/biotin system, this strategy can be extended for biosensor development as well as for other applications which require large surface patterning of proteins.

\section{Acknowledgments}

This study was funded by European Institute of Membrane (health project (PS1-2015)). V. F. has fellowship From UE Backis program.

The authors declare that they have no conflict of interest.

\section{References}

Aswal, D., S. Lenfant, D. Guerin, J. Yakhmi and D. Vuillaume (2006). "Self assembled monolayers on silicon for molecular electronics." Analytica chimica acta 568(1): 84-108.

Bain, C. D., E. B. Troughton, Y. T. Tao, J. Evall, G. M. Whitesides and R. G. Nuzzo (1989). "Formation of monolayer films by the spontaneous assembly of organic thiols from solution onto gold." Journal of the American Chemical Society 111(1): 321-335.

Bain, C. D. and G. M. Whitesides (1989). "Modeling organic surfaces with self-assembled monolayers." Angewandte Chemie International Edition in English 28(4): 506-512.

Balme, S., P.-E. Coulon, M. Lepoitevin, B. Charlot, N. Yandrapalli, C. Favard, D. Muriaux, M. Bechelany and J. M. Janot (2016). "Influence of adsorption on proteins and amyloids detection by silicon nitride nanopore." Langmuir 32: 8916-8925.

Balme, S., J. M. Janot, P. Dejardin, E. N. Vasina and P. Seta (2006). "Potentialities of confocal fluorescence for investigating protein adsorption on mica and in ultrafiltration membranes." Journal of Membrane Science 284(1-2): 198-204.

Bechelany, M., P. Brodard, L. Philippe and J. Michler (2009). "Extended domains of organized nanorings of silver grains as surface-enhanced Raman scattering sensors for molecular detection." Nanotechnology 20(45): 455302.

Bishop, A. R. and R. G. Nuzzo (1996). "Self-assembled monolayers: Recent developments and applications." Current Opinion in Colloid \& Interface Science 1(1): 127-136.

Boal, A. K., F. Ilhan, J. E. DeRouchey, T. Thurn-Albrecht, T. P. Russell and V. M. Rotello (2000). "Selfassembly of nanoparticles into structured spherical and network aggregates." Nature 404(6779): 746748.

Bog, U., F. Brinkmann, S. F. Wondimu, T. Wienhold, S. Kraemmer, C. Koos, H. Kalt, M. Hirtz, H. Fuchs and S. Koeber (2015). "Densely Packed Microgoblet Laser Pairs for Cross-Referenced Biomolecular Detection." Advanced Science 2(10): 1500066. 
Chapman, R. G., E. Ostuni, S. Takayama, R. E. Holmlin, L. Yan and G. M. Whitesides (2000). "Surveying for surfaces that resist the adsorption of proteins." Journal of the American Chemical Society 122(34): 8303-8304.

Danov, K. D., B. Pouligny and P. A. Kralchevsky (2001). "Capillary forces between colloidal particles confined in a liquid film: the finite-meniscus problem." Langmuir 17(21): 6599-6609.

Deckman, H. W. and J. H. Dunsmuir (1982). "Natural Lithography." Applied Physics Letters 41(4): $377-$ 379.

Dubois, L. H. and R. G. Nuzzo (1992). "Synthesis, structure, and properties of model organic surfaces." Annual review of physical chemistry 43(1): 437-463.

Florence, A. T. (2004). "Issues in oral nanoparticle drug carrier uptake and targeting." Journal of drug targeting 12(2): 65-70.

Gudipati, C. S., J. A. Finlay, J. A. Callow, M. E. Callow and K. L. Wooley (2005). "The antifouling and fouling-release perfomance of hyperbranched fluoropolymer (HBFP)-poly (ethylene glycol)(PEG) composite coatings evaluated by adsorption of biomacromolecules and the green fouling alga ulva." Langmuir 21(7): 3044-3053.

Hamming, L. M. and P. B. Messersmith (2008). "Fouling resistant biomimetic poly (ethylene glycol) based grafted polymer coatings." Mater. Matters 3: 52.

Love, J. C., L. A. Estroff, J. K. Kriebel, R. G. Nuzzo and G. M. Whitesides (2005). "Self-assembled monolayers of thiolates on metals as a form of nanotechnology." Chemical reviews 105(4): 11031170.

Ma, H., J. Hyun, P. Stiller and A. Chilkoti (2004). "“Non-Fouling” Oligo (ethylene glycol)-Functionalized Polymer Brushes Synthesized by Surface-Initiated Atom Transfer Radical Polymerization." Advanced Materials 16(4): 338-341.

Mallon, C. T., B. Jose, R. J. Forster and T. E. Keyes (2010). "Protein nanopatterning and release from gold nano-cavity arrays." Chemical Communications 46(1): 106-108.

Mikhael, B., B. Elise, M. Xavier, S. Sebastian, M. Johann and P. Laetitia (2011). "New silicon architectures by gold-assisted chemical etching." ACS Applied Materials \& Interfaces 3(10): 38663873.

Obermeier, B., F. Wurm, C. Mangold and H. Frey (2011). "Multifunctional poly (ethylene glycol) s." Angewandte Chemie International Edition 50(35): 7988-7997.

Ostuni, E., R. G. Chapman, R. E. Holmlin, S. Takayama and G. M. Whitesides (2001). "A survey of structure-property relationships of surfaces that resist the adsorption of protein." Langmuir 17(18): 5605-5620.

Rogach, A., A. Susha, F. Caruso, G. Sukhorukov, A. Kornowski, S. Kershaw, H. Möhwald, A. Eychmüller and H. Weller (2000). "Nano-and Microengineering: 3-D Colloidal Photonic Crystals Prepared from Sub- $\mu \mathrm{m}$-sized Polystyrene Latex Spheres Pre-Coated with Luminescent Polyelectrolyte/Nanocrystal Shells." Advanced Materials 12(5): 333-337.

Rybczynski, J., M. Hilgendorff and M. Giersig (2003). Nanosphere lithography-Fabrication of various periodic magnetic particle arrays using versatile nanosphere masks. Low-Dimensional Systems: Theory, Preparation, and Some Applications, Springer: 163-172.

Sakamoto, S., L. Philippe, M. Bechelany, J. Michler, H. Asoh and S. Ono (2008). "Ordered hexagonal array of Au nanodots on Si substrate based on colloidal crystal templating." Nanotechnology 19(40): 405304.

Soteropulos, C. E., K. M. Zurick, M. T. Bernards and H. K. Hunt (2012). "Tailoring the protein adsorption properties of whispering gallery mode optical biosensors." Langmuir 28(44): 1574315750.

Taylor, Z. R., J. C. Keay, E. S. Sanchez, M. B. Johnson and D. W. Schmidtke (2012). "Independently Controlling Protein Dot Size and Spacing in Particle Lithography." Langmuir 28(25): 9656-9663.

Uchida, K., H. Otsuka, M. Kaneko, K. Kataoka and Y. Nagasaki (2005). "A reactive poly (ethylene glycol) layer to achieve specific surface plasmon resonance sensing with a high $\mathrm{S} / \mathrm{N}$ ratio: the substantial role of a short underbrushed PEG layer in minimizing nonspecific adsorption." Analytical chemistry 77(4): 1075-1080. 
Ulman, A. (1996). "Formation and structure of self-assembled monolayers." Chemical reviews 96(4): 1533-1554.

Velev, O. and E. Kaler (1999). "In situ assembly of colloidal particles into miniaturized biosensors." Langmuir 15(11): 3693-3698.

Wang, C., F. L. Yap and Y. Zhang (2005). "Micropatterning of polystyrene nanoparticles and its bioapplications." Colloids and Surfaces B-Biointerfaces 46(4): 255-260.

Washburn, A. L., M. S. Luchansky, A. L. Bowman and R. C. Bailey (2009). "Quantitative, label-free detection of five protein biomarkers using multiplexed arrays of silicon photonic microring resonators." Analytical chemistry 82(1): 69-72.

Wünsch, J. R. (2000). Polystyrene: Synthesis, production and applications, iSmithers Rapra Publishing. Zalipsky, S. and J. Milton Harris (1997). Introduction to chemistry and biological applications of poly (ethylene glycol). ACS Symposium Series, American Chemical Society. 